\title{
Effects of immunosuppression with tacrolimus and mycophenolate mofetil on renal histology and function in single kidney rats submitted to ischemia and reperfusion ${ }^{1}$
}

\author{
Paulo Henrique Goulart Fernandes Dias ${ }^{\mathrm{I}}$, Gabriel Augusto Oliveira ${ }^{\mathrm{II}}$, Fernando Goulart Fernandes Dias ${ }^{\mathrm{III}}$, Regina de Paula \\ Xavier Gomes ${ }^{\text {IV }}$, Renato Tambara Filhov ${ }^{\text {, Rogério de Fraga }}{ }^{\text {II }}$
}

DOI: http://dx.doi.org/10.1590/S0102-86502015002000007

IFellow Master degree, Postgraduate Program in Clinical Surgery, Federal University of Parana (UFPR), Curitiba-PR, Brazil. Conception, design, intellectual and scientific content of the study; technical and surgical procedures; acquisition, analysis and interpretation of data; manuscript writing.

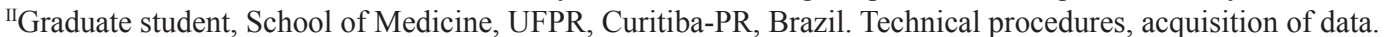

IIIMD, Urologist, Department of Urology, Campinas University (UNICAMP), Campinas-SP, Brazil. Interpretation of data, manuscript preparation and writing, critical revision.

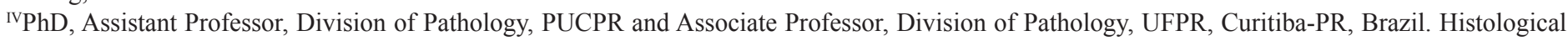
examinations, interpretation of data.

${ }^{\mathrm{v}} \mathrm{PhD}$, Full Professor, Division of Urology, Department of Surgery, UFPR, Curitiba-PR, Brazil. Design of the study, critical revision.

VIPhD, Assistant Professor, Division of Urology, Department of Surgery, UFPR, Curitiba-PR, Brazil. Conception, design, intellectual and scientific content of the study; critical revision.

\section{ABSTRACT}

PURPOSE: To evaluate renal histological changes and renal function in single kidney rats submitted to renal ischemia-reperfusion and to immunosuppression with tacrolimus and mycophenolate-mofetil.

METHODS: Experimental study with 80 Wistar rats distributed into control, Sham and six other groups treated with immunosuppressive drugs. Animals undergoing surgery, right nephrectomy and left renal clamping, killed on the $14^{\text {th }}$ day and analyzed for renal histology, urea and creatinine.

RESULTS: The group receiving tacrolimus at higher doses (T3) showed renal histological lesions indicative of early nephrotoxicity, and significant increase in urea and creatinine. The group M (mycophenolate-mofetil alone) and the group M2 (mycophenolate-mofetil combined with half the usual dose of tacrolimus) presented a slight rise in serum urea. The groups using mycophenolate-mofetil alone or combined with tacrolimus showed creatinine levels similar to that of the group T3.

CONCLUSIONS: Histologically, the association of injury by ischemia-reperfusion with the use of tacrolimus or mycophenolatemofetil alone demonstrated a higher rate of renal changes typical of early nephrotoxicity. In laboratory, the combination of injury by ischemia-reperfusion with tacrolimus at higher doses proved to be nephrotoxic.

Key words: Immunosuppression. Tacrolimus. Ischemia. Reperfusion. Histology. Kidney. Rats. 


\section{Introduction}

Nephropathy or chronic transplant dysfunction (NPC Tx) is the leading cause of kidney transplant failure, with slowly and progressive deterioration of renal function ${ }^{1}$. The multifactorial pathogenesis of NPC Tx is not yet fully understood, and immunological factors, inadequate immunosuppression and ischemia-reperfusion time of the organ play an important role in the pathophysiology $y^{2-4}$.

Currently, calcineurin inhibitors like tacrolimus and cyclosporine are the cornerstones of immunosuppressive therapy. These agents are administered combined with antiproliferative agents such as mycophenolate-mofetil (MMF), sirolimus and azathioprine, with or without steroids. The use of tacrolimus associated with MMF is considered the gold standard in most transplant programs in the United States ${ }^{5}$.

Much has been investigated about clinical immunosuppression, however, regardless of treatment regimen, the immunosuppressant drugs have adverse effects including nephrotoxicity ${ }^{5}$, cardiovascular effects ${ }^{5}$, diabetes mellitus ${ }^{5}$, cosmetic effects such as hirsutism, gum hyperplasia, alopecia ${ }^{5}$, and changes in the wound healing process ${ }^{6}$. Nephrotoxicity is a major clinical obstacle related to immunosuppression with calcineurin inhibitors and is often responsible for the discontinuation of treatment ${ }^{5}$. The histological features of nephrotoxicity induced by these drugs are severe interstitial fibrosis, usually in stripes, peritubular calcification, arteriolar hyalinosis and focal glomerulosclerosis, these changes may result in irreversible chronic renal failure in patients undergoing renal transplantation ${ }^{5}$.

The ischemic time of the transplanted kidney is also noteworthy among the risk factors for the development of NPC Tx, with studies showing that a longer period of ischemia results in release of cytokines, significantly higher vascular and glomerular changes associated with increased risk of chronic rejection ${ }^{4}$.

Injury induced by ischemia and reperfusion has been studied as a risk factor for the development of post-transplant renal dysfunction. Studies have shown that rats undergoing transplantation with longer time of ischemia-reperfusion showed significantly higher vascular and glomerular changes than animals submitted to transplantation with shorter time of ischemia-reperfusion ${ }^{7}$. Moreover, the authors observed that the release of cytokines that accompanies the recovery phase of ischemic injury can trigger the cascade of events that would lead to chronic rejection ${ }^{7}$.

In this context, there are few studies assessing the simultaneous effect of drug-induced nephrotoxicity and of ischemia and reperfusion on post-transplant renal dysfunction ${ }^{8}$. The model used in this trial aimed to evaluate the renal histological changes and renal function in Wistar rats with single kidney, subjected to ischemia and reperfusion and immunosuppression with tacrolimus and MMF.

\section{Methods}

This project was approved by the Ethics Committee on Animal Experimentation, Biological Sciences Department, Federal University of Parana (UFPR) (Certificate 386b). Ethical principles for animal experimentation of the Brazilian College of Animal Experimentation (COBEA) and the requirements set by the Guide for the care and use of experimental animals (Canadian Council on Animal Care) were observed.

\section{Technical procedures and postoperative}

We used eighty male albino Wistar rats (Rattus norvegicus albinus, Rodentia: Mammalia), weighing between 200 and 350 grams, with free access to water and food. Animals were randomly divided into eight groups (Table 1), the control group (C) did not undergo surgery. The other seven groups underwent surgery following aseptic technique and subsequent treatment (Table 1).

TABLE 1 - Groups of animals, surgical procedure and post-surgical treatment.

\begin{tabular}{lccc}
\hline GROUPS & N & $\begin{array}{c}\text { SURGICAL } \\
\text { PROCEDURE }\end{array}$ & TREATMENT \\
\hline C & 10 & No & $0.9 \%$ Saline \\
SHAM & 8 & Yes & $0.9 \%$ Saline \\
T1 & 10 & Yes & Tacrolimus $1 \mathrm{mg} / \mathrm{kg} / \mathrm{day}$ \\
T2 & 10 & Yes & Tacrolimus $0.1 \mathrm{mg} / \mathrm{kg} / \mathrm{day}$ \\
T3 & 9 & Yes & Tacrolimus $10 \mathrm{mg} / \mathrm{kg} /$ day \\
M1 & 10 & Yes & MMF 20mg $/ \mathrm{kg} / \mathrm{day}$ \\
MT1 & 11 & Yes & MMF 20mg $/ \mathrm{kg} / \mathrm{day}$ \\
& & & +Tacrolimus $1 \mathrm{mg} / \mathrm{kg} /$ day \\
MT2 & 10 & Yes & MMF 20mg/kg/day \\
& & & +Tacrolimus $0.5 \mathrm{mg} / \mathrm{kg} /$ day \\
\hline
\end{tabular}

C: control; Tacrolimus: Prograf ${ }^{\grave{O}}$, Janssen-Cilag Ltda.; MMF: Mycophenolate mofetil, CellCept ${ }^{\circledR}$, Genentech Roche.

Thirty minutes before the procedure we used intramuscular diazepam as pre-anesthetic at a dose of $5 \mathrm{mg} / \mathrm{kg}$. Anesthesia was induced by intraperitoneal injection of ketamine

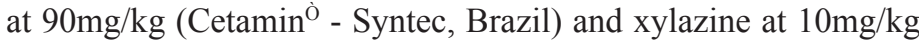

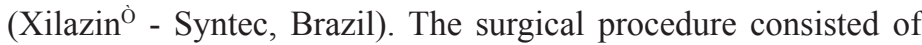


median laparotomy, right nephrectomy and vascular clamping of vessels of the pedicle of the left kidney for a period of 20 minutes (Figure 1). After this period of warm ischemia, the clamp was removed to allow reperfusion. Finally, we performed abdominal wall closure in layers.

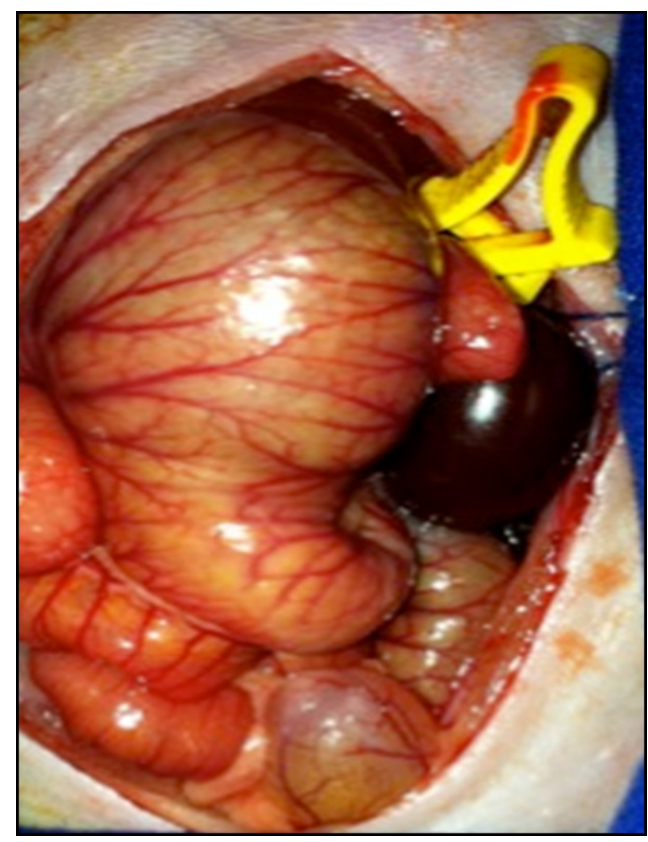

FIGURE 1 - Clamping of the left kidney.

On the first day after surgery, the animals received intramuscular analgesics (dipyrone) and daily, each group received a different type of solution by gavage with suitable technique and appropriate size of the tube (Table 1).

\section{Biochemical and histological analysis}

The animals were killed on day 14 of the experiment, under the same anesthesia protocol. Median thoracotomy was performed, followed by cardiac puncture with $25 \times 8 \mathrm{~mm}$ needle for taking blood sample, and induction of cardiac arrest by exsanguination. Samples were analyzed for serum tacrolimus, by chemiluminescence, and for urea and creatinine, by enzymatic colorimetric method. Subsequently, a new laparotomy was carried out with left nephrectomy and preparation of the organ for histological analysis. Kidneys were preserved in $10 \%$ formalin and routinely processed for histology and stained by immunohistochemical techniques of hematoxylin-eosin (HE), Masson's trichrome and periodic acid-Schiff (PAS).

The analysis and anatomopathological interpretation were performed using light microscopy. The following parameters were investigated: acute tubular necrosis (ATN); acute tubular toxicity (ATT), represented by cytoplasmic vacuolation; acute vascular toxicity (AVT), represented by arteriolar thrombosis (AT), glomerular thrombosis (GT) and intimal edema (IE); chronic toxicity (CT), represented by hyaline arteriolopathy (HA), tubular atrophy (TA), interstitial fibrosis (IF) and glomerular sclerosis (GS), and other less specific findings including inflammatory infiltrate (II) and hyaline casts (HC). These parameters were graded semi-quantitatively: absent, mild, moderate and severe, and transformed into quantitative variables by assigning numerical values, from 0 (absent) to 3 (severe). The histological score was calculated by averaging the indices of each group.

\section{Statistical analysis}

The serum levels of tacrolimus, creatinine and urea were analyzed separately, with calculation of mean, median and standard deviation of each group. To compare the levels of serum tacrolimus and urea, we used the Student's t-test. For the analysis of creatinine values, we applied the nonparametric Mann-Whitney test. Histological changes were analyzed using the histological score. For comparison between groups, the nonparametric MannWhitney test was used, with significance level of $95 \%(\mathrm{p}<0.05)$.

\section{Results}

Three rats died during the operative procedure, thus were excluded from the study. All animals were kept under the same care during the experiment, and none had complications or died during the postoperative observation.

\section{Biochemical and statistical analysis}

Serum levels of tacrolimus were higher according to the administered dose; in SHAM and control groups we did not detect serum levels of this drug. There was significant difference $(\mathrm{p}<0.05)$ in serum tacrolimus of the group T3 compared to the other groups (Figure 2A). The groups T1 and T2 showed difference in serum levels of tacrolimus, but it was not statistically significant $(\mathrm{p}=$ 0.084). The groups MT1 and MT2 had serum levels of tacrolimus statistically similar to groups T1 and T2.

Serum levels of urea were significantly greater in groups that received drugs compared to SHAM and control groups, except for the group M1, which exhibited no significant difference in relation to SHAM (M1xSHAM: $\mathrm{p}=0.19$ ) (Figure 2B). The group T3 demonstrated a significant increase of urea in comparison 
with all other groups $(\mathrm{p}<0.05)$. The group receiving MMF alone showed a significantly lower rate of urea increase compared to all other groups using tacrolimus, except for the group MT2 (half the usual dose of tacrolimus) (MxMT2: $\mathrm{p}=0.28$ ).

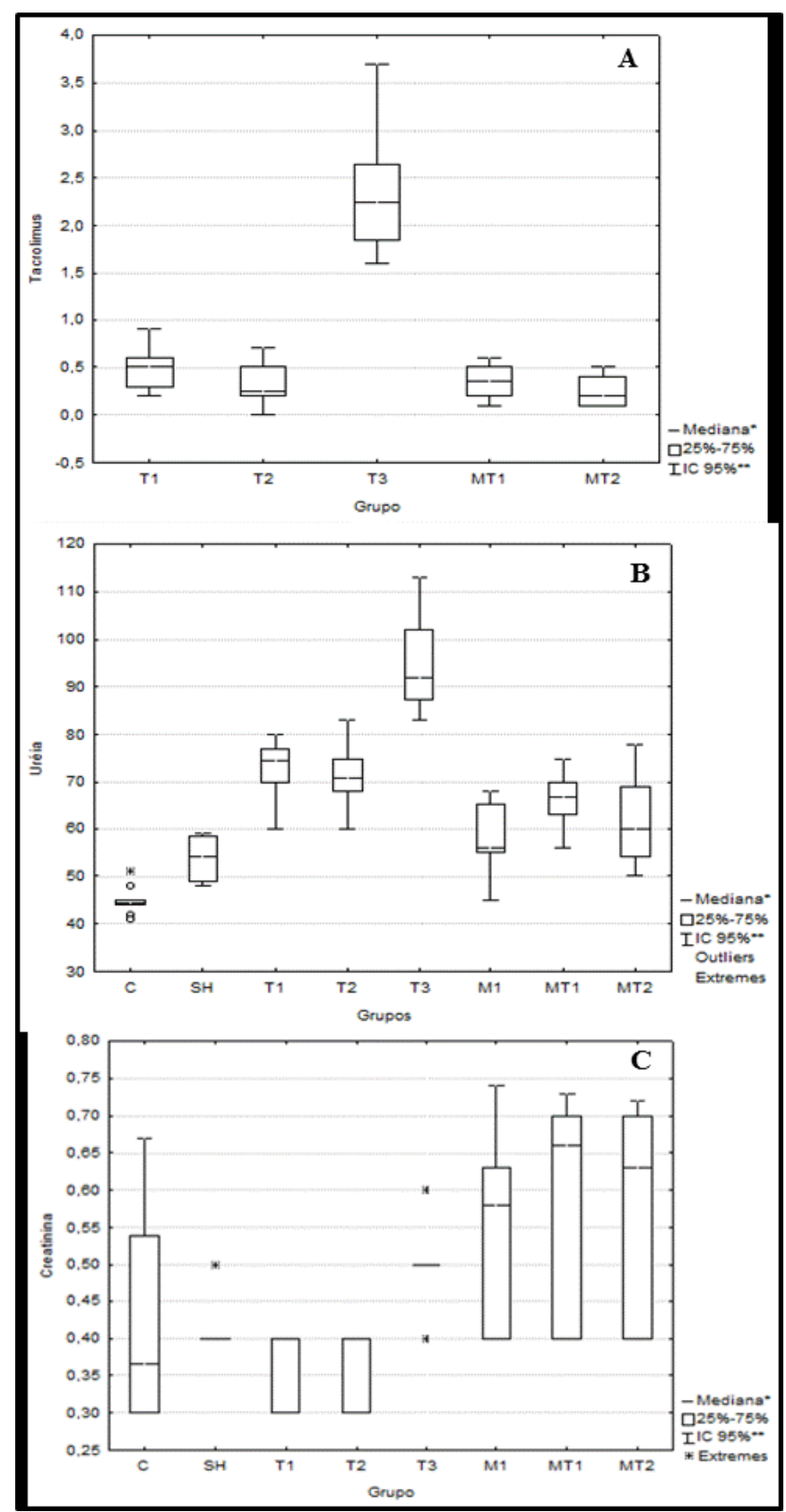

FIGURE 2 - Median and Standard Deviation of biochemical parameters analyzed. A. Serum levels of tacrolimus (ng/dl), control and SHAM groups did not show serum levels of this drug; B. Serum levels of urea; $\mathbf{C}$. Serum levels of creatinine.

Creatinine measurements showed no statistically significant differences between the groups control, SHAM, T1 and T2 with each other (Figure 2C). However, when creatinine values of the group T3 were compared to those of groups control, SHAM, T1 and T2, these differences were statistically significant.
The group in which MMF was used alone and those groups in which MMF was combined with tacrolimus showed serum levels of creatinine significantly higher when compared with all other groups, except for the group T3, to which a higher dose of tacrolimus was administered $(\mathrm{p}>0.05)$.

\section{Histological and statistical analysis}

There was no change suggestive of acute tubular necrosis (ATN). Likewise, no significant difference was evidenced between groups for arteriolar thrombosis, glomerular thrombosis and intimal edema (characteristics of acute vascular toxicity); interstitial fibrosis, arteriolopathy, glomerular sclerosis, tubular atrophy and glomerular hypertrophy (characteristics of chronic toxicity) and inflammatory infiltrate.

On the other hand, analyzing the cytoplasmic vacuolation of proximal tubules (Figure 3A), typical of acute tubular toxicity (ATT), we found a statistical difference between the group T3 with the control group $(\mathrm{p}=0.003)$ and a trend towards significance compared to SHAM ( $\mathrm{p}=0.056$ ). MMF, when administered alone, showed the highest score for cytoplasmic vacuolation (score of 0.8) (Figure 4), with significant difference compared to the groups control and SHAM. In turn, the group MT1 presented no difference compared to the group SHAM $(p=0.06)$ and the group MT2 showed a low score (0.20), quite similar to the groups SHAM $(p=0.83)$ and control.

Regarding the presence of hyaline casts, a statistical difference was observed between the group $\mathrm{T} 3$ and all other groups $(p=0.01)$; only the rats of this group showed these histological changes (Figure 3B).

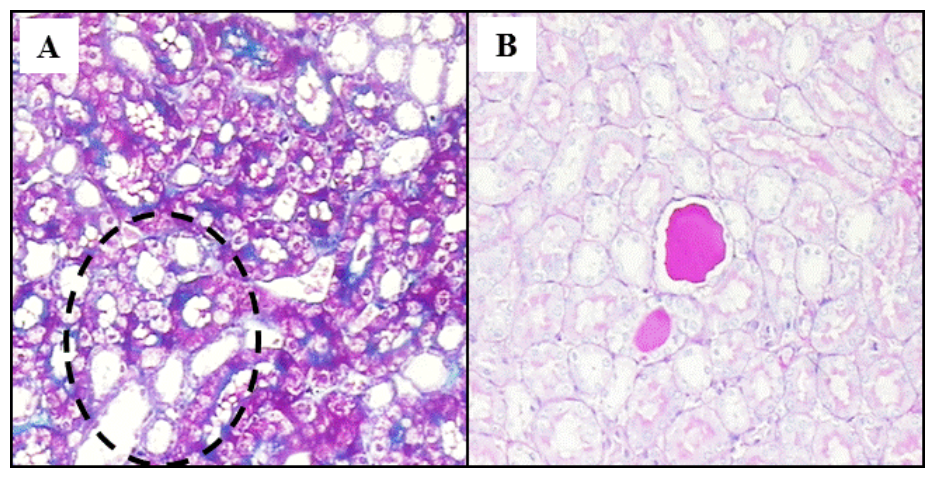

FIGURE 3 - Histological analysis. A. Cytoplasmic vacuolation (Masson trichrome staining, x40 Magnification). B. Hyaline casts (PAS staining, x100 Magnification). 


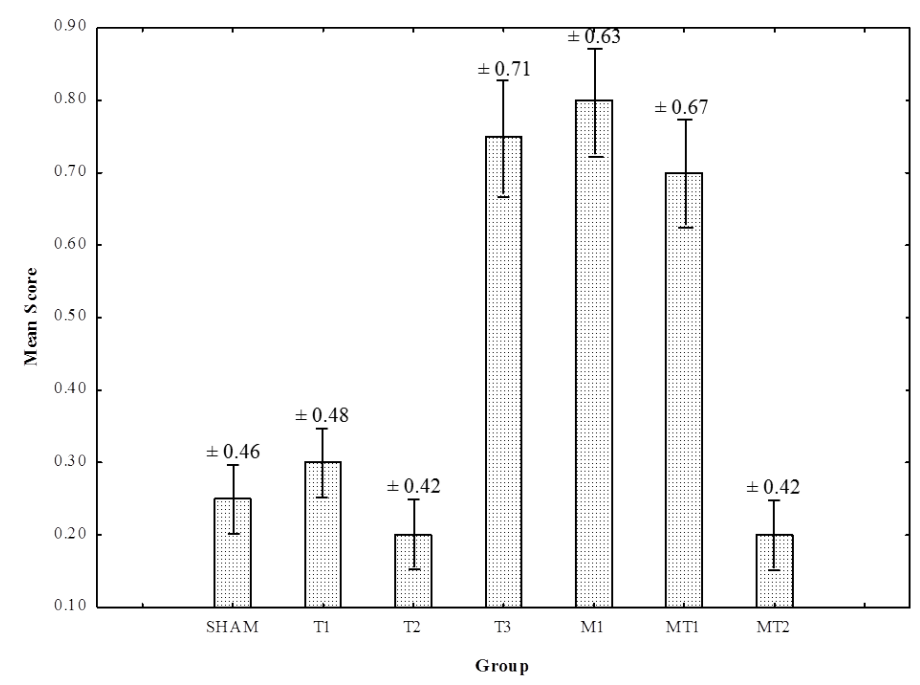

FIGURE 4 - Cytoplasmic vacuolation score in the tested groups (mean \pm standard deviation).

\section{Discussion}

New drugs have been developed and much has been studied on the best immunosuppressive regimen for renal transplantation. Achieving lower and lower rejection rates with different combinations of immunosuppressive drugs, the importance of safety and tolerability has become increasingly important, especially in relation to preservation of graft function and graft and patient survival ${ }^{5}$. Several Brazilian renal transplant services employ the combination of MMF and tacrolimus for immunosuppression, so the present experiment studied these drugs alone and in combination, and performed a stratification of their doses.

The ischemia-reperfusion injury model and the drug administration by gavage used in this experiment have already been described. Jain et al. ${ }^{1}$ studied the effects of tacrolimus and cyclosporine, submitted Wistar rats to clamping of the right kidney, associated with left nephrectomy and orally administered cyclosporine $(10 \mathrm{mg} / \mathrm{kg} /$ day $)$ or tacrolimus $(0-2 \mathrm{mg} / \mathrm{kg} /$ day $)$ through gavage. In the present study, we performed right nephrectomy and clamping of the left kidney because the vessels of the left renal hilum are longer, which facilitate clamping.

In this study, we used doses of tacrolimus between 0.1 $\mathrm{mg} / \mathrm{kg}$ and $10 \mathrm{mg} / \mathrm{kg}$ in order to observe the effects of medication from a sub-dose until high doses. According to Shihab et al. ${ }^{9}$, high doses of tacrolimus $(0.5-2.0 \mathrm{mg} / \mathrm{kg} /$ day $)$ are required for transplantation in rodents to prevent graft rejection, and the toxic therapeutic ratio between efficacy and side effects appears to be similar to that of humans.
Researches in animal models have shown that the consequences of ischemia-reperfusion depend on the duration of the procedure. In humans, ischemia during partial nephrectomy is associated with increased rates of acute renal failure and chronic kidney disease, and the maximum tolerated time, respectively for hot and cold renal ischemia is 20 and 35 minutos ${ }^{10}$. However, there is no consensus in the literature about the maximum period of ischemia-reperfusion to prevent irreversible renal damage ${ }^{11}$. In this way, we chose an ischemia-reperfusion period of 20 minutes, because prolonged periods can result in very severe kidney damage and even cause early death of animals.

The score model calculated by the average degree of histological findings used in this experiment had already been described. Nielsen et al. ${ }^{12}$ conducted an experiment aimed at evaluating glomerular function and renal morphological changes in rats treated with cyclosporine and tacrolimus via gavage, and sirolimus intraperitoneally. The morphological analysis of kidneys on the basis of a semi-quantitative score system evaluated subcapsular fibrosis and tubular basophilia. The tubular basophilia varied discretely in the treatment groups, on the other hand, fibrosis scores showed minimal variance. Shihab et al. ${ }^{9}$ also applied the score system for histological analysis through an experimental model developed with rats subjected to low salt diet and treated with subcutaneous tacrolimus ( $1 \mathrm{mg} / \mathrm{kg} /$ day) or to equivalent doses of placebo, killing the animals with seven or 28 days. Histological changes were examined by semi-quantitative score for each change (tubular injury, tubulointerstitial fibrosis and hyalinosis of afferent arterioles). Injuries typical of chronic nephrotoxicity caused by tacrolimus, such as interstitial fibrosis and arteriolopathy, were verified only at 28 days $^{9}$.

With regard to histological changes, this study showed mild changes. Study time of 14 days may have been decisive for the lack of evidence of lesions typical of chronic nephrotoxicity, as was demonstrated by Shihab et al. ${ }^{9}$, in which these lesions were not apparent after seven days, but only after 28 days. In the same way, Nielsen et al..$^{12}$, in the study period of 14 days showed minimal variance in the scores of fibrosis, as previously described. The most remarkable histological findings in this study were related to hyaline casts and cytoplasmic vacuolation of proximal tubules, characteristic of early nephrotoxicity. The first findings were evident only at high doses of tacrolimus. Cytoplasmic vacuolation was identified at higher scores in groups under high doses of tacrolimus and in the group in which MMF was used alone. The combination between MMF and tacrolimus at usual dose did not potentiate the vacuolation score, but, when combined with half the usual dose of tacrolimus, the score was 
below, similar to the SHAM group and statistically lower than the groups T3 and M1. This result suggests a possible protective effect of the combination of drugs.

Several studies investigated the impact of the use of immunosuppressive drugs on renal function. Nielsen et $a l .{ }^{12}$ registered that the use of cyclosporine, tacrolimus and sirolimus caused a significant decrease in creatinine clearance. In turn, Shihab et al. ${ }^{9}$ demonstrated that tacrolimus induced a significant increase in serum creatinine after 28 days of treatment. Considering the combination of drugs, prospective, randomized studies were carried out comparing immunosuppressive regimens on the basis of the use of tacrolimus combined with MMF and with sirolimus, and indicated that tacrolimus combined with MMF improves renal graft function and reduces cardiovascular risk, preventing and reducing the deterioration of graft function by chronic nephropathy ${ }^{13}$.

The present results demonstrated peculiar changes in renal function. The analysis of serum urea suggested a dose-dependent nephrotoxic effect of tacrolimus and a possible protective effect of this drug combined with MMF, corroborating previous studies ${ }^{13}$. On the other hand, high doses of tacrolimus significantly increased serum creatinine levels when compared with the control group and the SHAM group but lower doses did not cause significant changes. These results may be due to the fact that only the group T3 (mean $=2.35 ; \mathrm{SD}= \pm 0.67)$ presented serum levels of tacrolimus near the therapeutic range of the drug $(5-15 \mathrm{ng} / \mathrm{ml})^{14,15}$.

The reason for not having achieved therapeutic serum levels, even having been administered high doses of tacrolimus, seems to be related to the bioavailability of the drug. Oral administration of tacrolimus has low bioavailability ${ }^{9}$. Studies have found that subcutaneous administration may achieve serum levels of the therapeutic window for humans ${ }^{9}$ Besides that, these studies evidenced a high clearance of tacrolimus and higher volume of distribution in rats $(2.0 \mathrm{~L} / \mathrm{hr} / \mathrm{kg}$ and $20.2 \mathrm{~L} / \mathrm{kg}$, respectively) in comparison with the pharmacokinetics in humans $(0.1 \mathrm{~L} / \mathrm{hr} / \mathrm{kg}$ and $1 \mathrm{~L} / \mathrm{Kg}$, respectively), suggesting the use of higher doses in rats to reach the same serum levels ${ }^{9}$.

In this study, groups using MMF or MMF combined with tacrolimus showed significantly higher serum creatinine compared with all other groups, except for the group T3, in which a higher dose of tacrolimus was used ( $\mathrm{p}>0.05$ ). Such findings may result from a sub-dose of tacrolimus in the groups T1, T2, MT1 and MT2. As emphasized previously, only the group T3 presented serum levels of tacrolimus close to the therapeutic range of the drug. Another important issue is that the mean values of creatinine in groups M1, MT1 and MT2 were respectively 0.55, 0.59 and
0.58 , which were within normal reference standards of creatinine in rats $(0.6 \pm 0.02 \mathrm{mg} / \mathrm{dl})^{16}$.

\section{Conclusions}

Histologically, the association of injury by ischemiareperfusion with the use of tacrolimus or mycophenolate-mofetil alone demonstrated a higher rate of renal changes typical of early nephrotoxicity. In laboratory, the combination of injury by ischemia-reperfusion with tacrolimus at higher doses proved to be nephrotoxic.

\section{References}

1. Jain S, Bicknell GR, Nicholson ML. Tacrolimus has less fibrogenic potential than cyclosporin A in a model of renal ischemia-reperfusion injury. Br J Surg. 2000;87:1563-8. PMID: 11091246.

2. Tullius SG, Tilney NL. Both alloantigen-dependent and -independent factors influence chronic allograft rejection. Transplantation. 1995;59(3):313-8. PMID: 7871557.

3. Johnson CP, Kuhn EM, Hariharan S, Hartz AJ, Roza AM, Adams MB. Pre-transplant identification of risk factors that adversely affect length of stay and charges for renal transplantation. Clin Transplant. 1999;13(2):168-75. PMID: 10202613.

4. Bia MJ. Nonimmunologic causes of late renal graft loss. Kidney Int. 1995;47(6):1470-80. PMID: 7637276.

5. Gaston RS. Current and evolving immunosuppressive regimens in kidney transplantation. Am J Kidney Dis. 2006;47(4):S3-21. PMID: 16567239.

6. Ekici Y, Emiroglu R, Ozdemir H, Aldemir D, Karakayali H, Haberal M. Effect of rapamycin on wound healing: an experimental study. Transplat Proc. 2007;39(4):1201-3. PMID: 17524932.

7. Yilmaz S, Paavonen T, Hayry P. Chronic rejection of rat renal allografts. II. The impact of prolonged ischemia time on transplant histology. Transplatation. 1992;53(4):823-82. PMID: 1566348.

8. Ninova D, Covarrubias M, Rea DJ, Park WD, Grande JP, Stegall MD. Acute nephrotoxicity of tacrolimus and sirolimus in renal isografts: differential intragraft expression of transforming growth factor-[beta]1 and [alpha]-smooth muscle actin. Transplantation. 2004;78(3):338-44. PMID: 15316360.

9. Shihab FS, Bennett WM, Tanner AM, Andoh TF. Mechanism of fibrosis in experimental tacrolimus nephrotoxicity. Transplantation. 1997;64(12):1829-37. PMID: 9422427.

10. Thompson RH, Lane BR, Lohse CM, Leibovich BC, Fergany A, Frank I, Gill IS, Campbell, SC, Blute ML. Comparison of warm ischemia versus no ischemia during partial nephrectomy on a solitary kidney. Eur Urol. 2010;58:331-6. PMID: 20557996.

11. Meyer F, Silva DS, Bombonatto GM, Lizana JN, Dziedricki LF, Krieger ML. Análise histológica e do fluxo sanguímeo em rins submetidos a períodos de isquemia/reperfusão. Acta Cir Bras. 2011;26(6):451-5. PMID: 22042107.

12. Nielsen FT, Ottosen P, Starklint H, Dieperink H. Kidney function and morphology after short-term combination therapy with cyclosporine A, tacrolimus and sirolimus in the rat. Nephrol Dial Transplant. 2003;18(3):491-6. PMID: 12584269.

13. Sampaio EL, Pinheiro-Machado, PG, Garcia R, Felipe CR, Park SI, Casarini DE, Moreira S, Franco MF, Tedesco-Silva HJr, MedinaPestana JO. Mycopenolate mofetil vs. sirolimus in kidney transplant recipients receiving tacrolimus-based immunosuppressive regimen. 
Clin Transpl. 2008;22(2):141-9. PMID: 18339132.

14. Laskow DA. An open-label, concentration-ranging trial of FK506 in primary kidney transplantation: a report of the United States Multicenter FK506 Kidney Transplant Group Transplantation. Transplantation. 1996;62:900-5. PMID: 8878381.

15. Oellerich M. Therapeutic drug monitoring of cyclosporine and tacrolimus. Update on Lake Louise Consensus Conference on cyclosporine and tacrolimus. Clin Biochem. 1998;31:309-16. PMID: 9721427.

16. Castro BBA, Colugnati FAB, Cenedeze MA, Suassuna PGA, Pinheiro HS. Padronização da avaliação da função renal de ratos (Rattus norvegicus) Wistar do biotério da Universidade Federal de Juiz de Fora. J Bras Nefrol. 2014;36(2):139-149. doi: 10.5935/01012800.20140023 .

\section{Correspondence:}

Paulo Henrique Goulart Fernandes Dias

Av. Tiradentes 1319/zona 4

87013-260 Maringá - PR Brasil

Tel.: (55 44)3266-1508

paulohenriquegfd@yahoo.com.br

Received: Oct 15, 2014

Review: Dec 17, 2014

Accepted: Jan 19, 2015

Conflict of interest: none

Financial source: none

${ }^{1}$ Research performed at Department of Surgery, Federal University of Parana (UFPR), Curitiba-PR, Brazil. Part of Master degree thesis, Postgraduate Program in Clinical Surgery, UFPR. Tutor: Rogério de Fraga. 